3. I. N. Herstein and J. E. Adney, A note on the automorphism group of a finite group, Amer. Math. Monthly vol. 59 (1952) pp. 309-310.

4. W. R. Scott, On the order of the automorphism group of a finite group, Proc. Amer. Math. Soc. vol. 5 (1954) pp. 23-24.

5. H. Zassenhaus, The theory of groups (English trans.), New York, 1949.

6. W. Burnside, Theory of groups, Cambridge, 1897.

The Ohio State University

\title{
A NOTE ON FINITE UNIONS OF IDEALS AND SUBGROUPS
}

NEAL H. MCCOY

1. Introduction. From the theory of ideals in a commutative ring $R$ it follows easily that an ideal $I$ of $R$ is contained in the (settheoretic) union of a finite number of prime ideals $P_{i}(i=1,2, \cdots, n)$ of $R$ if and only if $I$ is contained in some one of the ideals $P_{i}$. A simple direct proof of this will be found in [2, p. 186]. Recently, Behrens $[1$, p. 171] has shown that the same result holds for the case in which neither commutativity nor associativity is assumed in $R$.

It is easy to see that if an ideal $I$ of a ring $R$ is contained in the union $A_{1} \cup A_{2}$ of any two ideals $A_{1}$ and $A_{2}$, it must be contained in one of them. For suppose that $I \subseteq A_{1} \cup A_{2}$ and that $I \nsubseteq A_{1}$. Then there exists an element $a_{2}$ of $I \cap A_{2}$ such that $a_{2} \notin A_{1}$. If $x \in I \cap A_{1}$, then $x+a_{2} \notin A_{1}$ and therefore $x+a_{2} \in A_{2}$ and $x \in A_{2}$. That is, $I \cap A_{1} \subseteq A_{2}$ and we have $I \subseteq A_{2}$. As a matter of fact, this result remains valid if $I, A_{1}$, and $A_{2}$ are subgroups of an arbitrary group. These observations were pointed out to me by Bailey Brown who also raised several questions about possible generalizations, some of which are partially answered in this note.

The following simple example, due to R. E. Johnson, shows that the above result about the union of two ideals no longer holds when we pass to the union of three ideals. Let $R$ be the ring whose additive group is the direct sum of two two-element cyclic groups, with every products equal to zero. Thus the elements of $R$ may be written as $(0,0),(0,1),(1,0)$, and $(1,1)$ with componentwise addition modulo 2. Then $A_{1}=\{(0,0),(0,1)\}, A_{2}=\{(0,0),(1,0)\}$, and $A_{3}=\{(0,0)$, $(1,1)\}$ are ideals in $R$, and $R$ is contained in the union of these three

Received by the editors September 30, 1956. 
ideals but not contained in the union of any two of them. However, since $R^{2}=0$, it is clear that $R^{2}$ is contained in all three of these ideals. This illustrates the first theorem to be proved below.

Theorem 1. Let $I$ and $A_{i}(i=1,2, \cdots, n)$ be ideals in a ring $R$ such that $I \subseteq A_{1} \cup A_{2} \cup \ldots \cup A_{n}$. If $I$ is not contained in the union of any $n-1$ of the ideals $A_{i}$, there exists a positive integer $k$, depending on $n$, such that $I^{k} \subseteq A_{1} \cap A_{2} \cap \cdots \cap A_{n}$.

The second theorem involves only one operation, and applies to subgroups of an arbitrary group $G$ which we shall assume to be written additively. However, we do not assume that $G$ is necessarily abelian.

If $I$ is a subgroup of $G$, and $m$ a positive integer, we shall find it convenient to use the notation, $m I=\{m a ; a \in I\}$. As an example, if $G$ is the additive group of the ring of four elements introduced above, then $2 G=0$ and therefore $2 G$ is contained in $A_{1} \cap A_{2} \cap A_{3}$. This illustrates our second theorem as follows.

Theorem 2. Let $I$ and $A_{i}(i=1,2, \cdots, n)$ be subgroups of a group $G$ such that $I \subseteq A_{1} \cup A_{2} \cup \ldots \cup A_{n}$. If $I$ is not contained in the union of any $n-1$ of the subgroups $A_{i}$, there exists a positive integer $l$, depending on $n$, such that $l I \subseteq A_{1} \cap A_{2} \cap \cdots \cap A_{n}$.

2. Proof of Theorem 1 . We assume that $n \geqq 3$ since the hypotheses of either theorem can not be satisfied otherwise. Since $I \subseteq A_{1} \cup A_{2}$ $\cup \ldots \cup A_{n}$, then clearly $I=\left(I \cap A_{1}\right) \cup\left(I \cap A_{2}\right) \cup \ldots \cup\left(I \cap A_{n}\right)$. For the purposes of the proof, we shall henceforth assume, as we clearly may without loss of generality, that $I=A_{1} \cup A_{2} \cup \cdots \cup A_{n}$. For convenience of notation, we shall also set $K=A_{1} \cap A_{2} \cap \cdots \cap A_{n}$.

As a first step we prove the following lemma.

LEMma. The intersection of any $n-1$ of the ideals $A_{i}$ coincides with $K$.

It is only necessary to show that this intersection is contained in $K$. Since $I$ is not contained in the union of any $n-1$ of the ideals $A_{i}$, there exists an element $a_{n}$ of $A_{n}$ which is not in $A_{1} \cup A_{2} \cup \ldots \cup A_{n-1}$. If $x \in A_{1} \cap A_{2} \cap \cdots \cap A_{n-1}$, the element $x+a_{n}$ of $I$ can not be in $A_{i}(i=1,2, \cdots, n-1)$; hence $x+a_{n} \in A_{n}$. It follows that $x \in A_{n}$ and so $x \in K$, as required.

We next establish the theorem for the case in which $n=3$. Since $A_{1} \cup A_{2} \subseteq A_{1}+A_{2}$, the relation $I=A_{1} \cup A_{2} \cup A_{3}$ implies at once that $I \subseteq\left(A_{1}+A_{2}\right) \cup A_{3}$. Since now $I$ is contained in the union of two ideals, we know that it is contained in one of them. However, $I \nsubseteq A_{3}$, and 
hence we have $I \subseteq A_{1}+A_{2}$. Similar arguments show that $I \subseteq A_{1}+A_{3}$ and that $I \subseteq A_{2}+A_{3}$. It follows that

$$
I^{3} \subseteq\left(A_{1}+A_{2}\right)\left(A_{1}+A_{3}\right)\left(A_{2}+A_{3}\right) .
$$

But if the right side of this is multiplied out, each term consists of a product containing at least two different ideals $A_{i}(i=1,2,3)$. The lemma then shows that $I^{3} \subseteq K$, and this completes the proof for the case in which $n=3$.

The proof is now easily completed by induction on $n$, and we therefore assume the theorem for the case of fewer than $n$ ideals $A_{i}$. It follows as above that

$$
I \subseteq\left(A_{1}+A_{2}\right) \cup A_{3} \cup \ldots \cup A_{n} .
$$

Now $I \subseteq A_{1}+A_{2}$ or, by possibly omitting some of the ideals on the right side of (1), we have $I$ contained in the union of some $m<n$ ideals but not in the union of any $m-1$ of them. Since $I \Phi A_{3} \cup \ldots$ $\cup A_{n}$, one of the remaining ideals must be $A_{1}+A_{2}$. Hence, by our induction hypothesis, there exists a positive integer $k_{1}$ such that $I^{k_{1}} \subseteq A_{1}+A_{2}$. A similar argument applies for each pair of ideals $A_{i}$, $A_{j},(i \neq j)$. Hence there exists a positive integer $k$ such that

$$
I^{k} \subseteq \prod_{i<j}\left(A_{i}+A_{j}\right)
$$

Now if the product on the right is expanded, it becomes a sum of ideals each of which is a product containing at least $n-1$ different ideals $A_{i}$. The lemma then shows that $I^{k} \subseteq K$, completing the proof.

In general, we have not determined the smallest possible value for the integer $k$. For $n=3$, the example given in the introduction shows that $k \geqq 2$, while the proof above suggests the value $k=3$. By other methods, it is possible to show that for $n=3$, we can always choose $k=2$; while for $n=4, k$ need not be greater than 6 .

REMARK. If one does not assume associativity of multiplication in $R$, the conclusion of Theorem 1 needs only to be modified to state that some product of $I$ with itself is contained in $K$. The proof goes through with only trivial changes.

We now give some corollaries of Theorem 1.

Corollary 1. Let $I$ and $B_{i}(i=1,2, \cdots, n)$ be ideals in $R$ with $I \subseteq B_{1} \cup B_{2} \cup \cdots \cup B_{n}$, and $I$ not contained in any single ideal $B_{i}$. Then there exists a positive integer $k$ such that $I^{k}$ is contained in at least three of the ideals $B_{i}(i=1,2, \cdots, n)$.

This follows by taking a minimal set of ideals $B_{i}$ whose union con- 
tains $I$, and applying the theorem. Such a minimal set must contain at least three ideals since, otherwise, $I$ would be contained in some one ideal.

Corollary 2. If $B_{i}(i=1,2, \cdots, n)$ are ideals in $R$ such that $B_{1} \cup B_{2} \cup \ldots \cup B_{n}$ is an ideal, there exists a positive integer $k$ such that $\left(B_{1} \cup B_{2} \cup \ldots \cup B_{n}\right)^{k}$ is contained in some one of the ideals $B_{i}$.

To see this, let $I=B_{1} \cup B_{2} \cup \ldots \cup B_{n}$. If $I \subseteq B_{i}$ for some $i$, there is nothing to prove. Otherwise we apply the preceding corollary.

Before stating the next corollaries we recall that if the ideal $B$ is semi-prime, then $I^{k} \subseteq B$ implies that $I \subseteq B$.

Corollary 3. Let I and $B_{i}(i=1,2, \cdots, n)$ be ideals in $R$ such that $I \subseteq B_{1} \cup B_{2} \cup \ldots \cup B_{n}$, where at least $n-2$ of the ideals $B_{i}$ are semi-prime. Then $I$ is contained in some one of the ideals $B_{i}$.

Corollary 4. If at least $n-2$ of the ideals $B_{i}(i=1,2, \cdots, n)$ are semi-prime, then $B_{1} \cup B_{2} \cup \ldots \cup B_{n}$ is an ideal if and only if $B_{1} \cup B_{2} \cup \ldots \cup B_{n} \subseteq B_{j}$ for some $j, 1 \leqq j \leqq n$.

3. Proof of Theorem 2. Again, to simplify the notation, we assume that $I=A_{1} \cup A_{2} \cup \cdots \cup A_{n}$, and let $K=A_{1} \cap A_{2} \cap \cdots \cap A_{n}$. Since the proof of the lemma involves only additive properties of $R$ and does not use commutativity of addition, the lemma is still valid in the setting of this theorem.

Let $m$ be a fixed integer, $1<m \leqq n-1$, such that for a suitable choice of the positive integer $s$,

$$
\left.s \text { (intersection of any } m \text { subgroups } A_{i}\right) \subseteq K \text {. }
$$

The lemma shows that for $m=n-1$ this is true with $s=1$. We now prove that we can pass from $m$ to $m-1$. Accordingly, we consider any $m-1$ of the subgroups $A_{i}$, and for convenience of notation let us choose $A_{i}(i=1,2, \cdots, m-1)$. Let $x \in A_{1} \cap A_{2} \cap \cdots \cap A_{m-1}$, and $a_{m}$ an element of $A_{m}$ which is in no $A_{i}(i \neq m)$. We consider the following elements of $I$ :

$$
x+a_{m}, \quad 2 x+a_{m}, \cdots,(n-m+1) x+a_{m} .
$$

If any one of these elements is in $A_{m}$, say $t x+a_{m} \in A_{m}$, then $t x \in A_{m}$ and (2) shows that stx $\in K$. Suppose, now, that no element (3) is in $A_{m}$. Clearly, no one of these elements can be in $A_{1} \cup A_{2} \cup \ldots \cup A_{m-1}$, so each is in some one of the subgroups $A_{m+1}, \cdots, A_{n}$. Since there are $n-m$ of these subgroups and $n-m+1$ elements (3), at least two of these elements must be in the same subgroup, say $A_{m+1}$. Suppose, 
for example, that $t_{1} x+a_{m} \in A_{m+1}$ and $t_{2} x+a_{m} \in A_{m+1}$, with $t_{1}<t_{2}$ $\leqq n-m+1$. It follows that

$$
\left(t_{2} x+a_{m}\right)-\left(t_{1} x+a_{m}\right)=\left(t_{2}-t_{1}\right) x \in A_{m+1},
$$

and hence that $\left(t_{2}-t_{1}\right) x \in A_{1} \cap A_{2} \cap \cdots \cap A_{m-1} \cap A_{m+1}$. Then (2) shows that $s\left(t_{2}-t_{1}\right) x \in K$. Since no one of the integers $t, t_{1}, t_{2}$ exceeds $n-m+1$, we have in every case $s(n-m+1) ! x \in K$. Hence, under the assumption (2), we have shown that

(4) $s(n-m+1) !\left(\right.$ intersection of any $m-1$ subgroups $\left.A_{1}\right) \subseteq K$.

Since we know that (2) is true for $m=n-1$, it is clear that the reduction from $m$ to $m-1$ can be continued to yield the existence of a positive integer $l$ such that $l A_{i} \subseteq K(i=1,2, \cdots, n)$, and hence that $l I \subseteq K$. The proof is therefore completed.

Actually, the proof shows that $l$ can be chosen to be $2 ! 3 ! \cdots$ $(n-1)$ ! but, except for the case in which $n=3$, it is not known whether this is the minimum possible choice for $l$.

Corollary 1. Let $I$ and $B_{i}(i=1,2, \cdots, n)$ be subgroups of $a$ group $G$ such that $I \subseteq B_{1} \cup B_{2} \cup \ldots \cup B_{n}$. If $I$ is not contained in any single one of the subgroups $B_{i}$, then there exists a positive integer $l$ such that $I I$ is contained in at least three of the subgroups $B_{i}$ $(i=1,2, \cdots, n)$.

Corollary 2. If $B_{i}(i=1,2, \cdots, n)$ are subgroups of the group $G$ such that $B_{1} \cup B_{2} \cup \ldots \cup B_{n}$ is a subgroup, there exists a positive integer l such that $l\left(B_{1} \cup B_{2} \cup \ldots \cup B_{n}\right)$ is contained in some one of the subgroups $B_{i}$.

\section{REFERENCES}

1. E. A. Behrens, Zur additiven Idealtheorie in nichtassoziativen Ringen, Math. Zeit. vol. 64 (1956) pp. 169-182.

2. N. H. McCoy, Rings and ideals, Carus Mathematical Monographs, no. 8, 1948.

Smith College 